\title{
KEYNOTE-585: Phase III study of perioperative chemotherapy with or without pembrolizumab for gastric cancer
}

\author{
Yung-Jue Bang*,1, Eric Van Cutsem², Charles S Fuchs ${ }^{3}$, Atsushi Ohtsu ${ }^{4}$, Josep Tabernero ${ }^{5}$, \\ David H Ilson ${ }^{6}$, Woo Jin Hyung ${ }^{7}$, Vivian E Strong ${ }^{8}$, Thorsten Oliver Goetze ${ }^{9}$, Takaki \\ Yoshikawa ${ }^{10}$, Laura H Tang ${ }^{8}$, Peggy May Tan Hwang ${ }^{11}$, Nancy Webb,11, David Adelberg ${ }^{11}$ \& \\ Kohei Shitara ${ }^{4}$ \\ ${ }^{1}$ Seoul National University College of Medicine, 101 Daehak-ro, Ihwa-Dong, Seoul, Republic of Korea \\ ${ }^{2}$ University Hospitals Gasthuisberg Leuven \& KU Leuven, 49 Herestraat, Leuven 3001, Belgium \\ ${ }^{3}$ Yale Cancer Center and Smilow Cancer Hospital, 333 Cedar Street, New Haven, CT 06510, USA \\ ${ }^{4}$ National Cancer Center Hospital East, 6-5-1, Kashiwanoha, Kashiwa, Chiba 277-8577, Japan \\ ${ }^{5}$ Vall d'Hebron University Hospital \& Institute of Oncology (VHIO), Vall d'Hebron 119-129, 08035 Universitat Autònoma de \\ Barcelona, Barcelona, Spain \\ ${ }^{6}$ Memorial Sloan Kettering Cancer Center, 300 East 66th Street, BAIC 1031, New York, NY 10065, USA \\ ${ }^{7}$ Yonsei Cancer Hospital, Yonsei University Health System, 50-1 Yonsei-Ro Seodaemun-gu, Seoul 03722, Republic of Korea \\ ${ }^{8}$ Memorial Sloan Kettering Cancer Center, 1275 York Ave, New York, NY 10065, USA \\ ${ }^{9}$ Institute of Clinical Cancer Research, UCT University Cancer Center Frankfurt, Steinbacher Hohl 2-26, Frankfurt 60488, Germany \\ ${ }^{10}$ National Cancer Center Hospital, 5-1-1 Tsukiji, Chuo-ku, Tokyo 104-0045, Japan \\ ${ }^{11}$ Merck \& Co., Inc., 2000 Galloping Hill Road, Kenilworth, NJ 07033, USA \\ *Author for correspondence: Tel.: +82 22072 2390; Fax: +82 2762 9662; bangyj@snu.ac.kr \\ ${ }^{\ddagger}$ Author working under contract with PRA Health Sciences, Raleigh, NC, USA
}

Background: Surgical resection is the only curative treatment option for gastric cancer. Despite widespread adoption of multimodality perioperative treatment strategies, 5-year overall survival rates remain low. In patients with advanced gastric or gastroesophageal junction adenocarcinoma, pembrolizumab has demonstrated promising efficacy and manageable safety as monotherapy in previously treated patients and as first-line therapy in combination with cisplatin and 5-fluorouracil. Combining chemotherapy with pembrolizumab in the neoadjuvant/adjuvant setting may benefit patients with locally advanced, resectable disease. Aim: To describe the design and rationale for the global, multicenter, randomized, double-blind, Phase III KEYNOTE-585 study to evaluate the efficacy and safety of pembrolizumab plus chemotherapy compared with placebo plus chemotherapy as neoadjuvant/adjuvant treatment for localized gastric or gastroesophageal junction adenocarcinoma.

ClinicalTrials.gov: NCT03221426

First draft submitted: 1 August 2018; Accepted for publication: 11 December 2018; Published online: 19 February 2019

Keywords: adjuvant therapy $\bullet$ chemotherapy $\bullet$ gastric cancer $\bullet$ gastroesophageal junction cancer $\bullet$ immunotherapy - neoadjuvant therapy $\bullet$ pembrolizumab • surgery

Gastric cancer is the fifth most common malignancy worldwide and the third leading cause of cancer death in males, with 723,100 deaths in 2012 [1,2]. There is significant global variation in prevalence and mortality rate of gastric cancer, influenced by regional variations in risk factors such as Helicobacter pylori infection and diet [1,2]. More than $70 \%$ of gastric cancer cases occur in developing countries, and approximately $50 \%$ of all cases occur in eastern Asian countries [2]. Similarly, mortality rates are highest in eastern Asia and lowest in North America [2].

Surgical resection in patients with nonmetastatic disease remains the only curative treatment option for gastric cancer [3]. However, recurrence rates after resection remain high, and multimodality treatment strategies incorporating perioperative (neoadjuvant and/or adjuvant) chemotherapy or chemoradiation are generally recommended [4-6]. The goal of neoadjuvant therapy is to reduce tumor burden and increase the possibility of complete (R0) resection [3] and to induce maximum pathological remission because pathological complete response ( $\mathrm{pCR}$ ) has been shown to

Future 8 Medicine 
correlate with overall survival (OS) [7]. The goal of adjuvant therapy is to reduce microscopic disease and prevent recurrence [3]. There is no universally accepted standard regimen for adjuvant therapy. Preferred strategies are typically perioperative chemotherapy in Europe, adjuvant chemoradiation in USA and adjuvant chemotherapy in Asia [4-8]. In European studies, perioperative chemotherapy with triplet or doublet regimens that include a fluoropyrimidine and a platinum agent before and after surgery have been shown to improve R0 resection rates and OS compared with surgery alone [9-11]. More recently, the AIO FLOT4 study showed superiority of a perioperative regimen comprising 5-fluorouracil plus docetaxel plus oxaliplatin plus leucovorin (FLOT) before and after surgery over perioperative treatment with epirubicin plus cisplatin plus 5-fluorouracil or capecitabine (ECF/ECX) with respect to R0 resection rate, progression-free survival and OS [12]. Perioperative chemotherapy, as previously described, is now the conventional treatment strategy in Europe and USA. Adjuvant chemotherapy alone after D2 resection is the standard of care in Asia, whereas neoadjuvant therapy is still considered investigational, and several randomized studies are ongoing [6]. Results of Asian studies have shown improved OS with adjuvant chemotherapy after D2 resection comprising a fluoropyrimidine, with or without a platinum agent $[13,14]$.

Overall, use of multimodality perioperative treatment strategies have consistently improved OS in gastric cancer. Nevertheless, 5-year OS for patients with gastric cancer is low at approximately $31 \%$ across all stages (67\% for patients with localized disease and $31 \%$ for patients with regional spread) [15].

\section{Keynote-585 study}

Herein, we describe the design and rationale for the multicenter, randomized, double-blind, Phase III KEYNOTE585 study (ClinicalTrials.gov: NCT03221426) conducted to evaluate the efficacy and safety of pembrolizumab plus chemotherapy compared with placebo plus chemotherapy as neoadjuvant/adjuvant treatment for localized gastric or gastroesophageal junction adenocarcinoma.

\section{Background \& rationale}

$\mathrm{PD}-1$ is a negative costimulatory receptor that is mainly expressed on activated $\mathrm{T}$ cells, and it is critical in the termination of immune responses and prevention of autoimmunity $[16,17]$. Tumor cells frequently use the PD-1 pathway to evade immune surveillance $[16,17]$. Binding of PD-1 to its ligands, PD-L1 and PD-L2, inhibits effector T-cell function, resulting in suppression of antitumor immune response [16,17]. PD-L1 is frequently overexpressed in gastric cancer, but the role of PD-L1 expression as a prognostic factor in gastric cancer is controversial [18-20]. In a systematic review that included the published results of 15 studies, PD-L1 expression was identified as a negative prognostic factor for OS in 11 studies, a positive prognostic factor in three studies and of no prognostic relevance in one study [21]. Meta-analysis of these 15 studies demonstrated a negative prognostic effect of PD-L1 expression on OS in gastric cancer, but the analysis was limited by the heterogeneity of the included studies [21]. Use of different PD-L1 antibodies, nonstandardized methods for assessing PD-L1 expression and relatively small sample sizes might have contributed to these inconsistent findings. In patients with stage II/III gastric cancer who underwent curative gastrectomy, PD-1 and PD-L1 expression were associated with worse disease-free survival (DFS) and OS [22].

The humanized, immunoglobulin G4 monoclonal antibodies nivolumab and pembrolizumab are immune checkpoint inhibitors that prevent interaction between the PD-1 and its ligands, PD-L1 and PD-L2 [23,24], and have demonstrated antitumor activity in multiple solid tumors. Use of nivolumab recently demonstrated a survival benefit compared with placebo (median OS 5.26 vs 4.14 months; hazard ratio: 0.63 ; 95\% CI: $0.51-$ 0.78; $\mathrm{p}<0.0001$ ) in the ATTRACTION-2 study of patients with advanced gastric or gastroesophageal junction cancer [25]. Pembrolizumab demonstrated promising antitumor activity and manageable safety as monotherapy in previously treated patients with metastatic gastric or gastroesophageal junction adenocarcinoma in the KEYNOTE012 and KEYNOTE-059 studies [26,27], resulting in approval by the US FDA for use in patients with a PD-L1 combined positive score (CPS) $\geq 1$ whose disease progressed on or after $\geq 2$ prior lines of therapy [23]. More recently, pembrolizumab monotherapy did not meet the protocol-specified criteria for a significant improvement in OS compared with paclitaxel as second-line therapy in patients with metastatic gastric or gastroesophageal cancer in the pivotal KEYNOTE-061 study [28]. When combined with cisplatin and 5-fluorouracil, pembrolizumab showed promising antitumor activity and manageable safety in patients with previously untreated metastatic gastric or gastroesophageal junction cancer in the Phase II KEYNOTE-059 study (ClinicalTrials.gov: NCT03221426) [29]. The confirmed objective response rate was 60\% (95\% CI: 39-79), with a higher response rate in patients with a PD-L1 CPS $\geq 1$ (73\%; 95\% CI: 45-92) than in patients with a PD-L1 CPS <1 (38\%; 95\% CI: 9-76). The 
Table 1. Study treatments.

\begin{tabular}{|c|c|c|c|c|}
\hline Treatment & Dose & Frequency & Route of administration & Dosing time of each 3-week cycle \\
\hline Pembrolizumab & $200 \mathrm{mg}$ & Q3W & IV infusion & Day 1 of cycles $1-17$ \\
\hline Placebo (normal saline) & $\mathrm{N} / \mathrm{A}$ & Q3W & IV infusion & Day 1 of cycles $1-17$ \\
\hline - Cisplatin & $80 \mathrm{mg} / \mathrm{m}^{2 \ddagger}$ & Q3W & IV infusion & Day 1 of cycles $1-6$ \\
\hline - Capecitabine & $1000 \mathrm{mg} / \mathrm{m}^{2 \ddagger}$ & Q3W & Oral & BID days $1-14$ of cycles $1-6^{\S}$ \\
\hline \multicolumn{5}{|l|}{ FLOT backbone ${ }^{\dagger, I:}$} \\
\hline - Docetaxel & $50 \mathrm{mg} / \mathrm{m}^{2 \ddagger}$ & Q2W & IV infusion & $\begin{array}{l}\text { Days } 1 \text { and } 15 \text { of cycles } 1 \text { and } 4 \text {, day } 8 \text { of cycles } 2 \text { and } \\
5 \text { and day } 1 \text { of cycles } 3 \text { and } 6\end{array}$ \\
\hline - Oxaliplatin & $85 \mathrm{mg} / \mathrm{m}^{2 \ddagger}$ & Q2W & IV infusion & $\begin{array}{l}\text { Days } 1 \text { and } 15 \text { of cycles } 1 \text { and } 4 \text {, day } 8 \text { of cycles } 2 \text { and } \\
5 \text { and day } 1 \text { of cycles } 3 \text { and } 6\end{array}$ \\
\hline - 5-Fluorouracil & $2600 \mathrm{mg} / \mathrm{m}^{2 \ddagger}$ & Q2W & IV infusion & $\begin{array}{l}\text { Days } 1 \text { and } 15 \text { of cycles } 1 \text { and } 4 \text {, day } 8 \text { of cycles } 2 \text { and } \\
5 \text { and day } 1 \text { of cycles } 3 \text { and } 6\end{array}$ \\
\hline - Leucovorin & $200 \mathrm{mg} / \mathrm{m}^{2 \ddagger}$ & Q2W & IV infusion & $\begin{array}{l}\text { Days } 1 \text { and } 15 \text { of cycles } 1 \text { and } 4 \text {, day } 8 \text { of cycles } 2 \text { and } \\
5 \text { and day } 1 \text { of cycles } 3 \text { and } 6\end{array}$ \\
\hline \multicolumn{5}{|c|}{$\begin{array}{l}\text { †Choice of chemotherapy backbone to be decided by the investigator before randomization. } \\
\text { ¥Body surface area to be calculated per local guidance. } \\
\text { §If dosed later in the day, only one dose may be taken on day } 1 \text { of the cycle, followed by BID dosing on days 2-14, with the final dose taken on the morning of day } 15 . \\
\text { I If adequate safety is demonstrated with FLOT + pembrolizumab in the FLOT safety cohort. } \\
\text { BID: Twice daily; FLOT: 5-Fluorouracil + docetaxel + oxaliplatin + leucovorin; FP: 5-Fluorouracil + cisplatin; IV: Intravenous; Q2W: Every } 2 \text { week; Q3W: Every } 3 \text { week; XP: } \\
\text { Capecitabine + cisplatin. }\end{array}$} \\
\hline
\end{tabular}

median progression-free survival was 7 months (95\% CI: 6-11 months), and median OS was 14 months (95\% CI: 9 months to not estimable).

In addition to demonstrating clinical activity in advanced cancers, including gastric cancer, use of anti-PD-1/PDL1 therapies has shown promising preclinical activity in the neoadjuvant setting [29]. In preclinical mouse models of triple-negative breast cancer, treatment with neoadjuvant anti-PD-1 plus anti-CD137 monoclonal antibodies improved survival and increased the number of cancer antigen-specific effector lymphocytes relative to adjuvant therapy after primary tumor resection [30]. In clinical studies, promising antitumor activity has been observed with pembrolizumab in combination with standard chemotherapy in the neoadjuvant setting in patients with triple-negative breast cancer and squamous cell carcinoma of the head and neck [31-33]. Additionally, adjuvant pembrolizumab monotherapy improved recurrence-free survival compared with placebo in patients with resected melanoma [34].

\section{Study design}

KEYNOTE-585 is a global, multicenter, randomized, double-blind, Phase III study (Figure 1). Eligible patients will be randomly assigned in a 1:1 ratio to receive pembrolizumab $200 \mathrm{mg}$ or placebo (normal saline) by intravenous infusion every 3 weeks in combination with chemotherapy. Chemotherapy will consist of cisplatin plus 5-fluorouracil (FP) or cisplatin plus capecitabine (XP), according to investigator choice (Table 1). Randomization will be performed using an interactive voice/web response system and will be stratified by geographic region (Asia vs non-Asia), tumor stage (II vs III vs IVa) and chemotherapy backbone (FP/XP vs other [for future additions of novel chemotherapy regimens]). Patients and investigators will be blinded to pembrolizumab or placebo assignment; administration of chemotherapy will be open label.

Neoadjuvant therapy will be administered for three cycles, followed by preoperative imaging assessment to evaluate surgical resectability. If there is no evidence of metastatic disease and the tumor is considered surgically resectable, patients will undergo surgical resection within 3-6 weeks after the last cycle of neoadjuvant therapy. Patients who have complete surgical resection (R0 resection) will receive adjuvant therapy starting within 4-10 weeks after surgery for 14 cycles (three cycles of combination therapy, followed by 11 cycles of pembrolizumab or placebo monotherapy). Patients with microscopic or macroscopic residual disease (R1 or R2 resection) can receive radiation therapy in addition to protocol-specified adjuvant therapy. Patients who are unable to undergo surgery can continue protocol-planned adjuvant therapy with or without radiation therapy as extended neoadjuvant/palliative therapy.

Based on recent data from the AIO FLOT4 study [12], a protocol amendment was instituted to incorporate a separate safety cohort to evaluate 5-fluorouracil plus docetaxel plus oxaliplatin plus leucovorin (FLOT) as a 


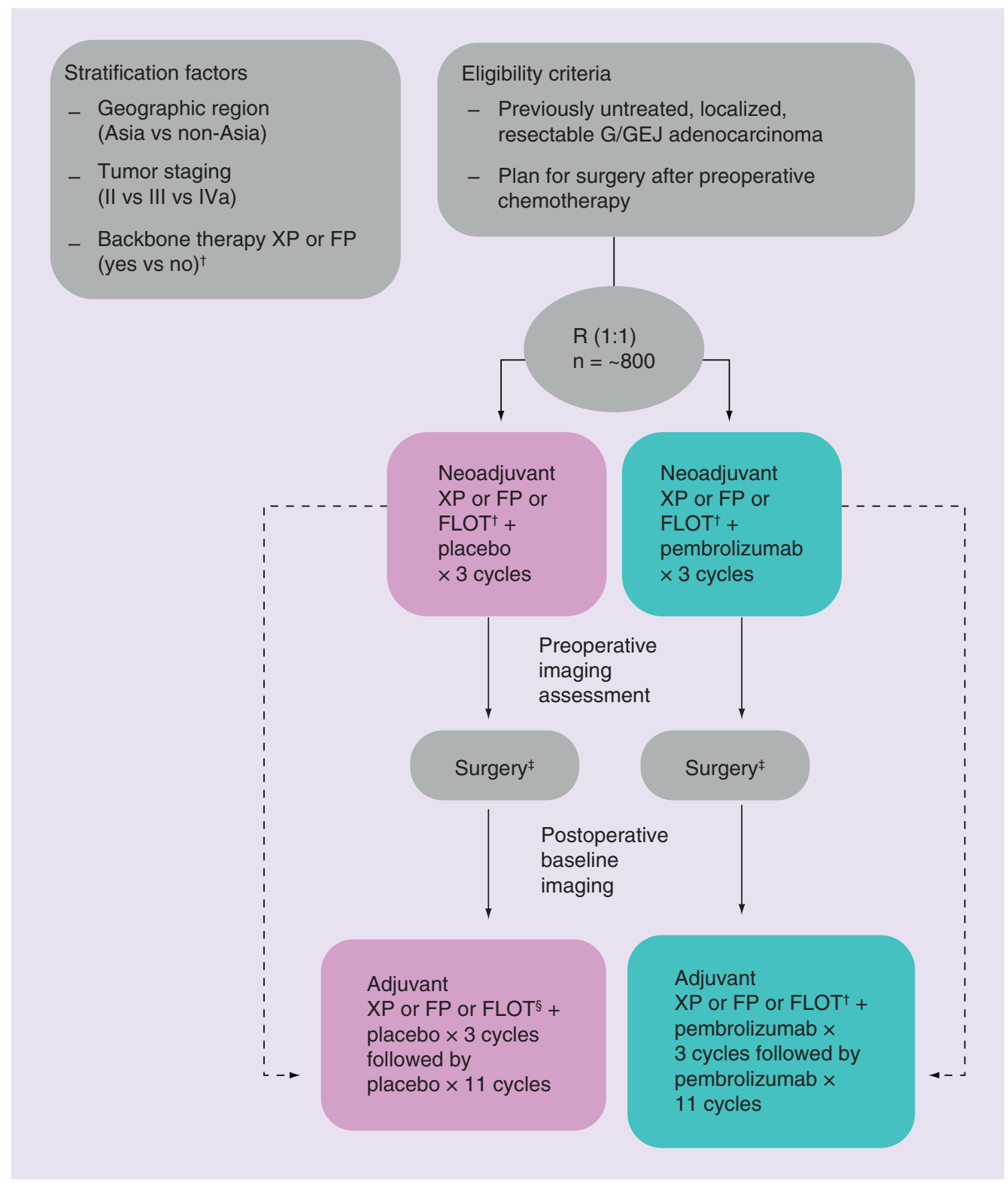

Figure 1. KEYNOTE-585 study design. Dotted line: patients unable to undergo surgery can move to adjuvant treatment after completing neoadjuvant treatment or undergo radiation therapy (in addition to adjuvant treatment). ${ }^{\dagger}$ If adequate safety is demonstrated with FLOT + pembrolizumab in the FLOT safety cohort.

¥Surgery will be performed within 3-6 weeks after the end of the last neoadjuvant cycle.

$\S$ Adjuvant therapy will begin within 4-10 weeks after surgery.

G/GEJ: Gastric/gastroesophageal junction; FLOT: 5-Fluorouracil + docetaxel + oxaliplatin + leucovorin; FP:

5-Fluorouracil + cisplatin; R: Randomization; XP: Capecitabine + cisplatin.

potential chemotherapy backbone option at selected study sites. In the FLOT safety cohort, eligible patients will be randomly assigned in a 1:1 ratio to receive pembrolizumab or placebo in combination with FLOT (Table 1), with stratification by tumor stage (II vs III vs IVa). Available safety data from the FLOT safety cohort will be reviewed by a standing internal data monitoring committee after patients have completed surgical resection and 30 days after surgical evaluation. After this review, the FLOT regimen might be incorporated as one of the chemotherapy backbone options in the main study if adequate safety is demonstrated in combination with pembrolizumab.

Eligibility criteria \& planned sample size \& study period

Eligibility criteria are described in Table 2 . The planned sample size is approximately 800 patients in the main 


\section{Inclusion criteria}

\section{- Males or females}

- Age $\geq 18$ years

- Previously untreated localized gastric or gastroesophageal junction

adenocarcinoma defined by $\mathrm{T} 3$ or greater primary lesion or the presence of any

positive clinical nodes without evidence of metastatic disease

- For gastroesophageal junction adenocarcinoma, Siewert type 2 or 3 tumors are

eligible (eligibility of Siewert type 1 tumors is limited to those for whom planned

treatment is perioperative chemotherapy and resection)

- Plan to proceed to surgery after preoperative chemotherapy

- Life expectancy $>6$ months

- Negative urine or serum pregnancy test within $72 \mathrm{~h}$ before the first dose of study treatment (females)

- Willing to use adequate contraception methods throughout the study and for 180 days after the last dose of chemotherapy (males and females), or 120 days

after the last dose of pembrolizumab, whichever is longer (females)

- Willing to provide tumor tissue at baseline and at the time of surgery

- Adequate hematological function, defined as $A N C \geq 1500 / \mu l$, platelet count

$\geq 100,000 / \mu \mathrm{l}$ and hemoglobin $\geq 9.0 \mathrm{~g} / \mathrm{dl}$ or $\geq 5.6 \mathrm{mmol} / \mathrm{l}$

- Adequate renal function, defined as creatinine $\leq 1.5 \times$ ULN, or measured or

calculated creatinine clearance $\geq 60 \mathrm{ml} / \mathrm{min}$ for those with creatinine levels

$>1.5 \times$ ULN

- Adequate hepatic function, defined as total bilirubin $\leq 1.5 \times$ ULN, or direct

bilirubin $\leq$ ULN for those with total bilirubin levels $>1.5 \times$ ULN and ALT/AST levels

$\leq 2.5 \times$ ULN

- Adequate coagulation function, defined as INR $\leq 1.5 \times$ ULN unless the patient is

receiving anticoagulant therapy with PT or APTT/PTT is within the therapeutic range

- Written informed consent
- ECOG performance status $0 / 1$

\section{Exclusion criteria}

- History of noninfectious pneumonitis that necessitated use of steroids or current pneumonitis

- Active infection necessitating systemic therapy

- Diagnosis of immunodeficiency

- Additional malignancy that is progressing or has necessitated active treatment

within the preceding 5 years (except for BCC or SCC of the skin, or carcinoma in situ that has undergone potentially curative therapy)

- Known hypersensitivity (grade $\geq 3$ ) to any of the study drugs or their excipients

- Active autoimmune disease that has necessitated systemic treatment (other than

replacement therapy) in the preceding 2 years

- Known history of HIV or HBV infection

- Known active HCV infection

- Known history of active tuberculosis

- Pregnant or breastfeeding, or expecting to conceive within the projected study duration

- History or current evidence of any condition, therapy or laboratory abnormality that might confound the study results or interfere with study participation

- Known psychiatric or substance abuse disorder that would interfere with cooperation with study requirements

- Prior treatment with anti-PD-1, anti-PD-L1 or anti-PD-L2 agent or an agent directed to another stimulatory or coinhibitory T-cell receptor (e.g., CTLA4, OX40 and CD137) - Prior systemic anticancer therapy (including investigational agents) for the current malignancy

- Prior radiotherapy within 2 weeks before the first dose of study treatment - Receiving long-term systemic steroid therapy $>10 \mathrm{mg}$ daily prednisone equivalent or any other form of immunosuppressive therapy within 14 days before the first dose of study treatment

- Received vaccination with live vaccine within 30 days before the first dose of study treatment

- Participation in a study of an investigational agent or device within 4 weeks before the first dose of study treatment

ALT: Alanine aminotransferase; ANC: Absolute neutrophil count; aPTT: Activated partial thromboplastin time; AST: Aspartate aminotransferase; BCC: Basal cell carcinoma; ECOG: Eastern Cooperative Oncology Group; HBV: Hepatitis B virus; HCV: Hepatitis C virus; HIV: Human immunodeficiency virus; INR: International normalized ratio; PT: Prothrombin time; PTT: Partial thromboplastin time; SCC: Squamous cell carcinoma; ULN: Upper limit of normal.

study and approximately 60 patients in the FLOT safety cohort. Assuming an average accrual rate of 30 patients per month, it is estimated that enrollment will take 27 months. The estimated time from enrollment of the first patient to the last patient's last visit is approximately 6 years.

\section{Outcome measures/end points}

The primary end points are OS, event-free survival (EFS) and pCR. OS is defined as the time from randomization to death from any cause. EFS is defined as the time from randomization to radiographic disease progression per Response Evaluation Criteria in Solid Tumors, version 1.1 (RECIST v1.1), by blinded independent central review, local or distant recurrence as assessed by computed tomography (CT) or biopsy if indicated (for participants who are disease free after surgery), or death from any cause; second primary malignancies are not considered EFS events. Pathological response will be graded according to Mandard criteria [35]; pCR is defined as no invasive disease within an entirely submitted and evaluated gross lesion and histologically negative nodes based on central review.

Secondary end points are safety and tolerability and DFS. DFS is defined as the time from the postsurgery baseline CT until the first documented occurrence of local or distant recurrence per RECIST v1.1 by blinded independent central review or death from any cause among patients who are disease free after surgery.

Exploratory end points include efficacy according to PD-L1 expression, R0 resection rate, health-related quality of life (assessed using the European Organisation for the Research and Treatment of Cancer Quality of Life Questionnaire [EORTC QLQ] core 30 items [C30] and gastric cancer-specific items [STO22]), utilities (assessed using the EuroQoL 5-dimension, 5-level questionnaire [EQ-5D-5L]) and molecular biomarkers.

\section{Study procedures}

Imaging assessments will be performed using CT or magnetic resonance imaging if CT is contraindicated. Initial tumor imaging will be performed during screening (within 21 days before randomization). The first on-study imaging assessment will be performed after completion of three cycles of preoperative therapy and before surgery (approximately 9 weeks [ \pm 7 days] after the first dose of study treatment or earlier if clinically indicated). Patients 
who undergo surgery will also undergo postsurgery baseline imaging within 2 weeks before the first dose of the adjuvant treatment. Subsequent imaging assessments will be performed every 12 weeks for the first 2 years and every 24 weeks thereafter. Primary evaluation of pathological response in surgically resected specimens will be performed by local pathologists, and assessments of pCR will be confirmed microscopically by a central pathologist. Patients will be followed up for survival status every 12 weeks until death, withdrawal of consent or end of study (whichever occurs first).

Health-related quality-of-life assessments (EORTC QLQ-C30, EORTC QLQ-STO22 and EQ-5D-5L) will be administered at baseline; day 1 of cycles 1 and 3 during neoadjuvant treatment; day 1 of cycles 1, 3, 4, 7, 10 and 14 during adjuvant therapy, at treatment discontinuation, and every 6 months for 3 years from randomization during the follow-up period. Safety will be monitored throughout the study and for 30 days after the end of treatment (90 days for serious adverse events), including adverse events, adverse events leading to discontinuation, serious adverse events, fatal adverse events, physical examination, vital signs, Eastern Cooperative Oncology Group performance status and laboratory values. Adverse events will be graded using National Cancer Institute Common Terminology Criteria for Adverse Events, version 4.0.

Tumor PD-L1 expression will be assessed by use of a Good Manufacturing Practice immunohistochemistry assay (PD-L1 IHC 22C3 pharmDx; Agilent Technologies, CA, USA). PD-L1 expression is measured as the CPS, or the number of PD-L1-positive cells (tumor cells, lymphocytes and macrophages) divided by the total number of tumor cells, multiplied by 100 . Additional exploratory biomarker analyses using tumor tissue and/or blood samples might include genetic analyses, mRNA expression profiling, proteomics and immunohistochemistry and other blood-derived biomarkers to identify novel predictive or pharmacodynamic markers of pembrolizumab in combination with chemotherapy

Statistics

Efficacy will be analyzed in the intent-to-treat population (all randomly assigned patients) by assigned treatment group. DFS will be evaluated in the subgroup of patients who are disease free at postsurgery baseline imaging. Safety will be analyzed in all randomly assigned patients who received at least one dose of study medication according to the treatment received.

The primary hypothesis for OS and EFS will be evaluated by comparing pembrolizumab plus chemotherapy with placebo plus chemotherapy using a stratified log-rank test. Hazard ratios will be estimated using a stratified Cox regression model, and event rates over time will be estimated within each treatment group using the Kaplan-Meier method. pCR rates will be compared between the treatment groups using the Miettinen and Nurminen method. Interim analyses are planned. An external data monitoring committee will review efficacy and safety results to determine whether the study will continue according to prespecified criteria.

\section{Conclusion}

Given the strong evidence of antitumor activity observed with pembrolizumab in combination with chemotherapy in patients with advanced gastric cancer and evidence of activity for pembrolizumab in the neoadjuvant/adjuvant setting, a strong rationale exists to evaluate the role of pembrolizumab in combination with perioperative chemotherapy in patients with resectable gastric cancer. Herein, we described the methodology of the KEYNOTE-585 study, an ongoing Phase III study conducted to evaluate the efficacy and safety of pembrolizumab plus chemotherapy compared with placebo plus chemotherapy as neoadjuvant/adjuvant treatment for localized gastric or gastroesophageal junction adenocarcinoma. It is hoped that results from the KEYNOTE-585 study will elucidate the role of immunotherapy in the perioperative treatment of resectable gastric cancer.

Supplementary data

An infographic accompanies this paper at the end of the references section. To download the infographic that accompanies this paper, please visit the journal website at: www.futuremedicine.com/doi/full/10.2217/fon-2018-0581

Acknowledgements

The authors thank the patients and their families and caregivers for participating in the study. 
Financial \& competing interests disclosure

Funding for this research was provided by Merck Sharp \& Dohme Corp., a subsidiary of Merck \& Co., Inc., Kenilworth, NJ, USA. Y-J Bang has served in a consulting or advisory role for Samyang and BeiGene and has received research funding from AstraZeneca/Medlmmune, Novartis, Genentech/Roche, MSD, Merck Serono, Bayer, GlaxoSmithKline, Bristol-Myers Squibb, Pfizer, Lilly, Boehringer Ingelheim, Macrogenics, Takeda, Boston Biomedical, Five Prime Therapeutics, CKD, Ono, Taiho, BeiGene, Curis Pharma and Greencross Biotherapeutics. EV Cutsem has served in a consulting or advisory role for Bayer, Lilly, Roche and Servier, and has received research funding from Amgen, Bayer, Boehringer, Celgene, Lilly, MSD, Merck, Novartis, Ipsen, Roche, Sanofi and Servier. C Fuchs has served in a leadership role for CytomX and has served in a consulting or advisory role for Eli Lilly, Entrinsic Health, Genentech, Merck, Sanofi, Five Prime Therapeutics, Merrimack, Bayer, Agios, Taiho, Kew, Unum Therapeutics and Bain Capital. A Ohtsu has received research funding from Bristol-Myers Squibb and reports employment of an immediate family member with Celgene. J Tabernero has served in a consulting or advisory role for Amgen, Bayer, Boehringer Ingelheim, Celgene, Chugai, Genentech, Lilly, MSD, Merck Serono, Novartis, Pfizer, Roche, Sanofi, Symphogen, Taiho and Takeda. D Ilson has served in a consulting or advisory role for Pieris, AstraZeneca, Astellas, Bristol-Myers Squibb, Pfizer, Bayer, Lilly, Roche, Oncolys and Merck. WJ Hyung reports stock ownership in Hutom, has served in a consulting or advisory role for Ethicon, and has received research funding from Medtronics. V Strong reports no conflicts of interest. TO Goetze has served in a consulting or advisory role for MSD, Lilly, Shire, Celgene, Bayer and Bristol-Myers Squibb, has served on a speaker's bureau for Lilly, and has received reimbursement for travel, accommodation or expenses from Bristol-Myers Squibb and Lilly. T Yoshikawa has received honoraria from Chugai Pharma, Yakult, Taiho Pharmaceutical, Abbott Nutrition, Takeda, Daiichi Sankyo, Ajinomoto, Ono Pharmaceutical, Nihonkayaku, Johnson \& Johnson, Medtronic, Olympus and Lilly, has served in a consulting or advisory role for Novartis, MSD Oncology, and Chugai Pharma, and has received research funding from Novartis, Chugai Pharma, Taiho Pharmaceutical and Yakult. L Tang reports no conflicts of interest. PMT Hwang, N Webb and D Adelberg are employees of Merck Sharp \& Dohme Corp., a subsidiary of Merck \& Co., Kenilworth, NJ, USA. MT Hwang also reports stock ownership for Merck Sharp \& Dohme Corp., a subsidiary of Merck \& Co., Kenilworth, NJ, USA. K Shitara has received honoraria from Novartis, AbbVie and Yakult, has served in a consulting or advisory role from Astellas Pharma, Lilly, Bristol-Myers Squibb, Takeda, Pfizer and Ono Pharmaceutical, and has received research funding from Lilly, Ono Pharmaceutical, Dainippon Sumoitomo Pharma, Daiichi Sankyo, Taiho Pharmaceutical, Chugai Pharma and MSD. The authors have no other relevant affiliations or financial involvement with any organization or entity with a financial interest in or financial conflict with the subject matter or materials discussed in the manuscript apart from those disclosed.

Medical writing and/or editorial assistance was provided by M Sweetlove, and SS Shaevitz, of the ApotheCom pembrolizumab team (Yardley, PA, USA). This assistance was funded by Merck Sharp \& Dohme Corp., a subsidiary of Merck \& Co., Inc., Kenilworth, NJ, USA.

\section{Ethical conduct of research}

The authors state that they have obtained appropriate institutional review board approval and have followed the principles outlined in the Declaration of Helsinki for all human experimental investigations. In addition, informed consent has been obtained from the participants involved. 
Summary points

- Surgery is the only curative treatment option for localized gastric cancer.

- Despite the widespread adoption of perioperative treatment strategies, 5-year overall survival is poor.

Background \& rationale

- PD-L1 is overexpressed in gastric cancer.

- Pembrolizumab has shown promising efficacy and manageable safety as monotherapy in treatment-experienced patients and combined with cisplatin and 5-fluorouracil as first-line therapy in patients with advanced gastric or gastroesophageal junction adenocarcinoma.

- Combining chemotherapy with pembrolizumab in the neoadjuvant/adjuvant setting might be beneficial for patients with locally advanced, resectable disease.

KEYNOTE-585 study design \& eligibility criteria

- KEYNOTE-585 is a multicenter, randomized, double-blind, Phase III study to evaluate the efficacy and safety of pembrolizumab plus chemotherapy compared with placebo plus chemotherapy as neoadjuvant/adjuvant treatment of patients with localized gastric or gastroesophageal junction adenocarcinoma.

- Eligible patients with previously untreated localized gastric or gastroesophageal junction adenocarcinoma will be randomly assigned to receive pembrolizumab or placebo in combination with chemotherapy (cisplatin +5 -fluorouracil or capecitabine, according to investigator choice).

- A separate safety cohort will evaluate 5 -fluorouracil + docetaxel + oxaliplatin + leucovorin as a potential chemotherapy option that might be incorporated into the main study if adequate safety is demonstrated.

Outcome measures/end points

- The primary end points are overall survival, event-free survival and pathological complete response rate.

Conclusion

- The KEYNOTE-585 study will elucidate the role of immunotherapy in the perioperative treatment of patients with resectable gastric cancer.

\section{References}

Papers of special note have been highlighted as: $\bullet$ of interest; $\bullet \bullet$ of considerable interest

1. Torre LA, Bray F, Siegel RL, Ferlay J, Lortet-Tieulent J, Jemal A. Global cancer statistics, 2012. CA Cancer J. Clin. 65(2), 87-108 (2015).

2. Ferlay J, Soerjomataram I, Dikshit $\mathrm{R}$ et al. Cancer incidence and mortality worldwide: sources, methods and major patterns in GLOBOCAN 2012. Int. J. Cancer 136(5), e359-e386 (2015).

3. Van Cutsem E, Sagaert X, Topal B, Haustermans K, Prenen H. Gastric cancer. Lancet 388(10060), 2654-2664 (2016).

4. Smyth EC, Verheij M, Allum W, Cunningham D, Cervantes A, Arnold D. Gastric cancer: ESMO Clinical Practice Guidelines for diagnosis, treatment and follow-up. Ann. Oncol. 27(Suppl. 5), v38-v49 (2016).

5. National Comprehensive Cancer Network. NCCN clinical practice guidelines in oncology (NCCN guidelines): gastric cancer (Version 2.2018). May 22, 2018. www.nccn.org/professionals/physician_gls/pdf/gastric.pdf

6. Japanese Gastric Cancer Association. Japanese gastric cancer treatment guidelines 2014 (ver. 4). Gastric Cancer 20(1), 1-19 (2017).

7. Li Z, Shan F, Wang Y et al. Correlation of pathological complete response with survival after neoadjuvant chemotherapy in gastric or gastroesophageal junction cancer treated with radical surgery: a meta-analysis. PLoS ONE 13(1), e0189294 (2018).

8. Russo A, Li P, Strong VE. Differences in the multimodal treatment of gastric cancer: east versus west. J. Surg. Oncol. 115(5), 603-614 (2017).

9. Ychou M, Boige V, Pignon JP et al. Perioperative chemotherapy compared with surgery alone for resectable gastroesophageal adenocarcinoma: an FNCLCC and FFCD multicenter Phase III trial. J. Clin. Oncol. 29(13), 1715-1721 (2011).

10. Schuhmacher C, Gretschel S, Lordick F et al. Neoadjuvant chemotherapy compared with surgery alone for locally advanced cancer of the stomach and cardia: European Organisation for Research and Treatment of Cancer randomized trial 40954. J. Clin. Oncol. 28(35), 5210-5218 (2010).

11. Cunningham D, Allum WH, Stenning SP et al. Perioperative chemotherapy versus surgery alone for resectable gastroesophageal cancer. N. Engl. J. Med. 355(1), 11-20 (2006).

12. Al-Baltran SE, Pauligk C, Homann N et al. Docetaxel, oxaliplatin, and fluorouracil/leucovorin (FLOT) for resectable esophagogastric cancer: updated results from multicenter, randomized Phase 3 FLOT4-AIO trial (German Gastric Group at AIO). Ann. Oncol. 28(Suppl. 5), 616 (2017).

- The AIO FLOT4 study showed superiority of a perioperative regimen comprising 5-fluorouracil plus docetaxel plus oxaliplatin plus leucovorin (FLOT) before and after surgery over perioperative treatment with epirubicin plus cisplatin plus 5-fluorouracil or capecitabine (ECF/ECX) with respect to R0 resection rate, progression-free survival and overall survival. The results of this trial helped position perioperative chemotherapy as the conventional treatment strategy in Europe and USA. 
13. Sakuramoto S, Sasako M, Yamaguchi T et al. Adjuvant chemotherapy for gastric cancer with S-1, an oral fluoropyrimidine. N. Engl. J. Med. 357(18), 1810-1820 (2007).

14. Bang YJ, Kim YW, Yang HK et al. Adjuvant capecitabine and oxaliplatin for gastric cancer after D2 gastrectomy (CLASSIC): a Phase III open-label, randomised controlled trial. Lancet 379(9813), 315-321 (2012).

15. Howlader N, Noone AM, Krapcho M et al. SEER Cancer Statistics Review, 1975-2014. National Cancer Institute, MD, USA. https://seer.cancer.gov/csr/1975_2014/

16. Francisco LM, Sage PT, Sharpe AH. The PD-1 pathway in tolerance and autoimmunity. Immunol. Rev. 236, 219-242 (2010).

17. Pardoll DM. The blockade of immune checkpoints in cancer immunotherapy. Nat. Rev. Cancer 12(4), 252-264 (2012).

18. Geng Y, Wang H, Lu C et al. Expression of costimulatory molecules B7-H1, B7-H4 and Foxp ${ }^{3+}$ Tregs in gastric cancer and its clinical significance. Int. J. Clin. Oncol. 20(2), 273-281 (2015).

19. Hou J, Yu Z, Xiang R et al. Correlation between infiltration of FOXP ${ }^{3+}$ regulatory $\mathrm{T}$ cells and expression of B7-H1 in the tumor tissues of gastric cancer. Exp. Mol. Pathol. 96(3), 284-291 (2014).

20. Boger C, Behrens HM, Mathiak M, Kruger S, Kalthoff H, Rocken C. PD-L1 is an independent prognostic predictor in gastric cancer of western patients. Oncotarget 7(17), 24269-24283 (2016).

21. Gu L, Chen M, Guo D et al. PD-L1 and gastric cancer prognosis: a systematic review and meta-analysis. PLoS ONE 12(8), e0182692 (2017).

22. Eto S, Yoshikawa K, Nishi M et al. Programmed cell death protein 1 expression is an independent prognostic factor in gastric cancer after curative resection. Gastric Cancer 19(2), 466-471 (2016).

23. Merck Sharp \& Dohme Corp. KEYTRUDA ${ }^{\circledR}$ (pembrolizumab) injection, for intravenous use. Whitehouse Station, NJ, USA (2017).

24. OPDIVO (nivolumab) injection, for intravenous use [prescribing information]. Bristol-Myers Squibb, NJ, USA (2018).

25. Kang YK, Boku N, Satoh T et al. Nivolumab in patients with advanced gastric or gastro-oesophageal junction cancer refractory to, or intolerant of, at least two previous chemotherapy regimens (ONO-4538-12, ATTRACTION-2): a randomised, double-blind, placebo-controlled, Phase III trial. Lancet 390(10111), 2461-2471 (2017).

26. Muro K, Chung HC, Shankaran V et al. Pembrolizumab for patients with PD-L1-positive advanced gastric cancer (KEYNOTE-012): a multicentre, open-label, Phase IB trial. Lancet 17(6), 717-726 (2016).

- In this paper on the Phase IB KEYNOTE-012 study, pembrolizumab was reported to have demonstrated a $22 \%$ objective response rate in patients with PD-L1-positive advanced gastric or gastroesophageal junction adenocarcinoma. These data, along with subsequent data from the KEYNOTE-059 study (reference 27) led to the US FDA approval of pembrolizumab for the treatment of patients with recurrent or metastatic G/GEJ adenocarcinoma whose tumors express PD-L1 and whose disease has progressed on or after $\geq 2$ prior lines of therapy.

27. Fuchs CS, Doi T, Jang RW et al. Safety and efficacy of pembrolizumab monotherapy in patients with previously treated advanced gastric and gastroesophageal junction cancer: Phase 2 clinical KEYNOTE-059 trial. JAMA Oncol. 4(5), e180013 (2018).

-. In cohort 1 of the KEYNOTE-059 study, pembrolizumab monotherapy demonstrated durable responses, with higher ORR and longer response durations in patients with advanced gastric and gastroeosphageal cancer. Based on results from this and the KEYNOTE-012 study (reference 26), the FDA approved pembrolizumab for the treatment of patients with recurrent or metastatic G/GEJ adenocarcinoma whose tumors express PD-L1 and whose disease has progressed on or after $\geq \mathbf{2}$ prior lines of therapy.

28. Shitara K, Özgüroğlu M, Bang Y-J et al. Pembrolizumab versus paclitaxel for previously treated, advanced gastric or gastro-oesophageal junction cancer (KEYNOTE-061): a randomised, open-label, controlled, Phase III trial. Lancet 392(10142), 123-133 (2018).

29. Wainberg ZA, Jalal S, Muro K et al. KEYNOTE-059 update: efficacy and safety of pembrolizumab alone or in combination with chemotherapy in patients with advanced gastric or gastroesophageal (G/GEJ) cancer. Ann. Oncol. 28(Suppl. 5), 616 (2017).

30. Liu J, Blake SJ, Yong MC et al. Improved efficacy of neoadjuvant compared to adjuvant immunotherapy to eradicate metastatic disease. Cancer Discov. 6(12), 1382-1399 (2016).

31. Nanda R, Liu MC, Yau C et al. Pembrolizumab plus standard neoadjuvant therapy for high-risk breast cancer (BC): results from I-SPY 2. J. Clin. Oncol. 35(Suppl.), 506-506 (2017).

32. Schmid P, Hee Park Y, Muñoz-Couselo E et al. Pembrolizumab (pembro) + chemotherapy (chemo) as neoadjuvant treatment for triple negative breast cancer (TNBC): preliminary results from KEYNOTE-173. J. Clin. Oncol. 35(15 Suppl.), 556-556 (2017).

33. Uppaluri R, Zolkind P, Lin T et al. Neoadjuvant pembrolizumab in surgically resectable, locally advanced HPV negative head and neck squamous cell carcinoma (HNSCC). J. Clin. Oncol. 35(Suppl.), 6012-6012 (2017).

34. Merck's KEYTRUDA ${ }^{\circledR}$ (pembrolizumab) significantly improved recurrence-free survival compared to placebo as adjuvant therapy in patients with stage 3 resected high-risk melanoma (EORTC1325/KEYNOTE-054) [press release]. Business Wire, NJ, USA (2018).

35. Mandard AM, Dalibard F, Mandard JC et al. Pathologic assessment of tumor regression after preoperative chemoradiotheapy of esophageal carcinoma: clinicopathologic correlations. Cancer 73(11), 2680-2686 (1994). 


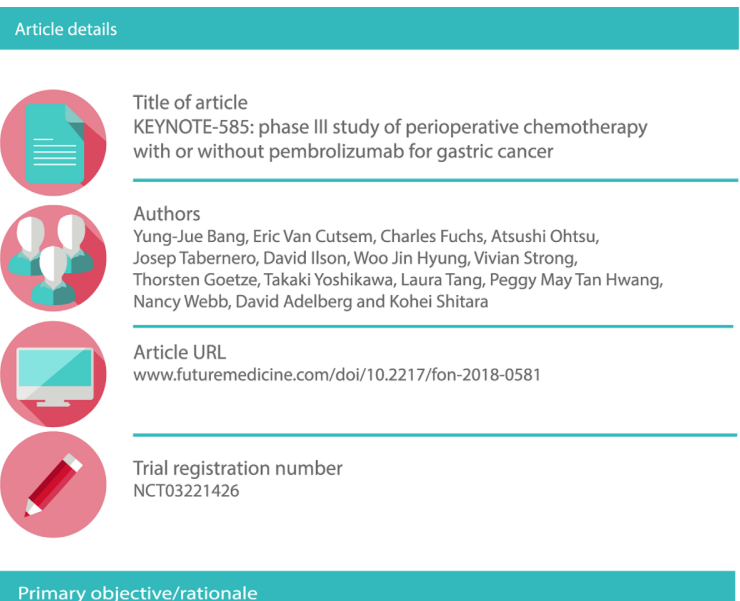

Primary objective

Evaluate $\mathrm{OS}, \mathrm{EFS}, \mathrm{pCR}$, and safety and tolerability of pembrolizumab plus chemotherapy compared with placebo plus chemotherapy as neoadjuvant and/or adjuvant treatment for localized gastric or gastroesophageal junction adenocarcinoma

\section{Secondary objectives}

Evaluate safety and tolerability and DFS of pembrolizumab plus chemotherapy compared with placebo plus chemotherapy as neoadjuvant and/or adjuvant treatment for localized gastric or gastroesophageal junction adenocarcinoma

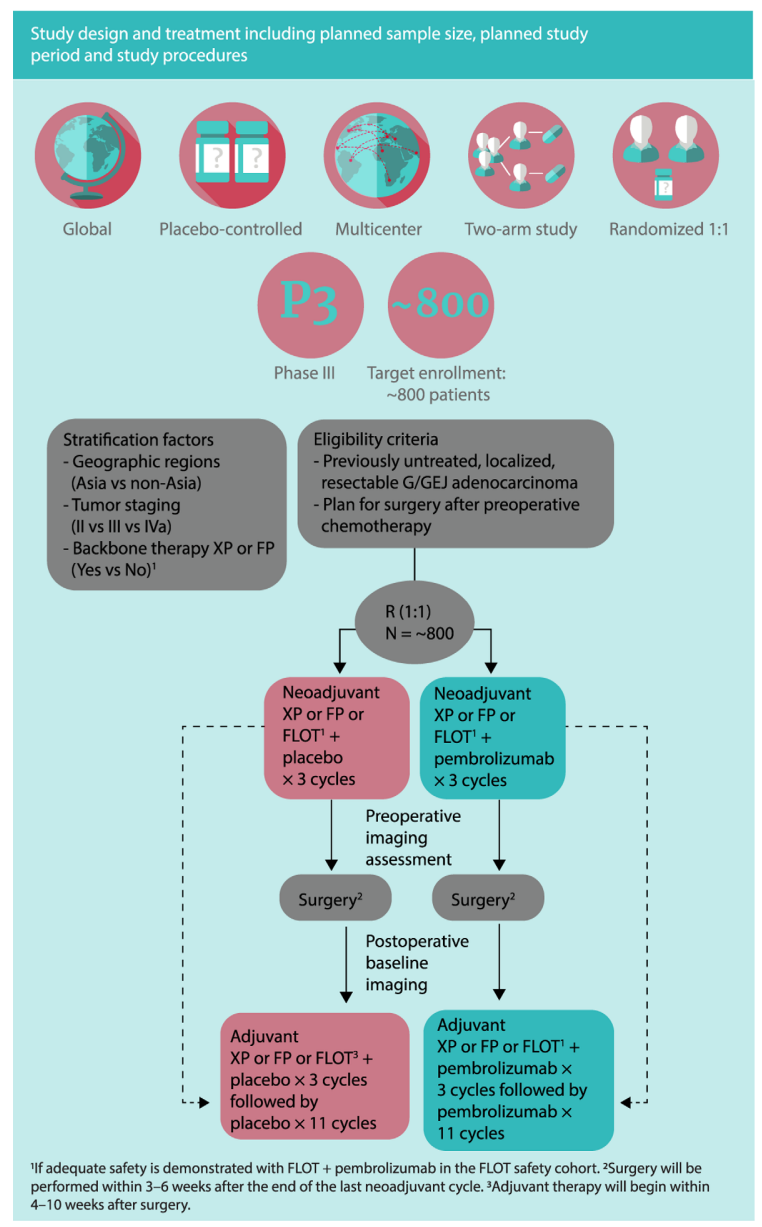

\section{Study design and treatment including planned sample size, planned study} period and study procedures (cont)

Receive chemotherapy + pembrolizumab (arm 1) or chemotherapy + placebo (arm 2). Patients will receive neoadjuvant (preoperative) chemotherapy + pembrolizumab Q3W for 3 cycles or chemotherapy + placebo Q3W for 3 cycles followed by surgery, then adjuvant chemotherapy + pembrolizumab Q3W for 3 cycles or chemotherapy + placebo Q3W for 3 cycles, then monotherapy with pembrolizumab or placebo Q3W for 11 cycles. Chemotherapy is cisplatin $80 \mathrm{mg} / \mathrm{m}^{2}$ iv. on day $1+$ either capecitabine $1000 \mathrm{mg} / \mathrm{m}^{2}$ orally twice daily $\times 14$ days or $5-\mathrm{FU} 800 \mathrm{mg} / \mathrm{m}^{2}$ iv. daily $\times 5$ days (investigator's choice). Pembrolizumab $200 \mathrm{mg}$ iv. is given on day 1. Adjuvant monotherapy is pembrolizumab (arm 1) or placebo (arm 2). In a separate safety cohort, 5 -FU $2600 \mathrm{mg} / \mathrm{m}^{2}$ iv. + docetaxel $50 \mathrm{mg} / \mathrm{m}^{2}$ iv. + oxaliplatin $85 \mathrm{mg} / \mathrm{m}^{2}$ iv. + leukovorin $200 \mathrm{mg} / \mathrm{m}^{2} \mathrm{iv}$. (FLOT) is being studied as a potential chemotherapy option

Stratified according to geographic region (Asia vs. non-Asia), tumor stage (II vs. III vs. IVa), and chemotherapy backbone (XP/FP versus other [for future additions of novel chemotherapy regimens])

Treatment will continue until confirmed radiographic progression, unacceptable toxicity, Treatment will continue until confirmed radiographic progression, unacceptable toxicity, or completion of 35 cycles of pembrolizumab or placebo (approximately 2 years)

\section{Key eligibility criteria}

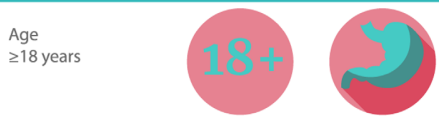

Previously untreated localized gastric or gastroesophageal junction adenocarcinoma defined by $\mathrm{T} 3$ or greater primary lesion or the presence of any positive clinical nodes without evidence of metastatic disease

For gastroesophageal junction adenocarcinoma, Siewert type 2 or 3 tumors are eligible (eligibility of Siewert type 1 tumors is limited to those for whom planned treatment is perioperative chemotherapy and resection)

Plan to proceed to surgery after preoperative chemotherapy

ECOG performance status $0 / 1$

No active autoimmune disease

Adequate organ function

Willing to provide tumor tissue at baseline and at the time of surgery

\section{Outcome measures/end points}

Primary end points

OS, defined as the time from randomization to death from any cause: EFS, defined as the time from randomization to radiographic disease progression per Response Evaluation Criteria in Solid Tumors, version 1.1 (RECIST v1.1), by blinded independent central review, local or distant recurrence as assessed by computed tomography (CT) or biopsy if indicated (for participants who are disease free after surgery), or death from any cause; and $\mathrm{pCR}$, defined as no invasive disease within an entirely submitted and evaluated gross lesion and histologically negative nodes based on central review; pathologic response will be graded according to Mandard criteria

Secondary end points

Safety and tolerability and DFS, defined as the time from the post-surgery baseline $C T$ until the first documented occurrence of loca or distant recurrence per RECIST 111 by blinded independent central review or death from any cause among patients who are disease free after surgery

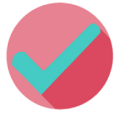

Exploratory end points

Efficacy according to PD-L1 expression, R0 resection rate, HRQO (assessed using the European Organisation for the Research and Treatment of Cancer Quality of Life Questionnaire [EORTC QLO] core 30 items [C30] and gastric cancer-specific items [STO22]), utilities (assessed using the EuroQoL 5-dimension, 5-level questionnaire [EQ-5D-5L]) and molecular biomarkers

\section{Glossary}

DFS: Disease-free survival; EFS: Event-free survival; G/GEJ: Gastric/gastroesophageal junction FLOT: 5-fluorouracil + docetaxel + oxaliplatin + leukovorin; FP: 5-fluorouracil + cisplatin OS: Overall survival; pCR: Pathologic complete response; PD-L1: Programmed death ligand 1 Q3W: Every 3 weeks; R: Randomization; XP: Capecitabine + cisplatin 